\title{
Factors associated with bone mineral density in adults: a cross-sectional population-based study
}

\author{
Fatores associados à densidade mineral óssea em adultos: \\ um estudo transversal baseado em população \\ Factores asociados con la densidad mineral ósea en adultos: \\ un estudio transversal basado en población
}

How to cite this article:

Segheto KJ, Juvanhol LL, Carvalho CJ, Silva DCG, Kakehasi AM, Longo GZ. Factors associated with bone mineral density in adults: a cross-sectional population-based study. Rev Esc Enferm USP. 2020;54:e03572. doi: https://doi.org/10.1590/S1980-220X2018039903572

\section{Kátia Josiany Segheto ${ }^{1}$}

Leidjaira Lopes Juvanhol ${ }^{1}$

Cristiane Junqueira de Carvalho ${ }^{1}$

Danielle Cristina Guimarães da Silva ${ }^{1}$

Adriana Maria Kakehasi ${ }^{2}$

Giana Zarbato Longo ${ }^{1}$

${ }^{1}$ Universidade Federal de Viçosa,

Viçosa, MG, Brazil.

${ }^{2}$ Universidade Federal de Minas Gerais, Belo Horizonte, MG, Brazil.

\begin{abstract}
Objective: This study aimed to analyze the association between lumbar spine, femoral neck, total hip bone mineral density (biophysical bone health assessment parameter), and sociodemographic, anthropometric, behavioral, and health condition factors in Brazilian adults. Method: This is a cross-sectional, population-based study performed with individuals of both genders, aged between 20 and $59(n=701)$. The dependent variables were evaluated by Dual Energy X-ray Absorptiometry. The independent variables were evaluated through a questionnaire, anthropometric evaluation and blood collection. The association between bone mineral density and the independent variables was evaluated by linear regression analysis. All analyses were stratified by gender. Results: Men presented higher bone mineral density than women. Bone mineral density was inversely associated with age range and directly associated with nutritional status in both genders and in the three bone sites analyzed. In addition, 25 Hydroxyvitamin D deficient status among men and contraceptive use among women were associated with lower bone mineral density, and a significant association was only found with lumbar spine bone mineral density in women. Conclusion: The factors associated with bone health among men were age, skin color, nutritional status, and vitamin $\mathrm{D}$ status. For women, the associated factors with bone health were age, skin color, nutritional status and contraceptive use.
\end{abstract}

DESCRIPTORS

Bone Density; Risk Factors; Adult Health; Epidemiologic Studies. 


\section{INTRODUCTION}

The world has undergone great socioeconomic, demographic and epidemiological changes in the last decades, which have caused (among other situations) a gradual increase in life expectancy. Brazil is not different from other countries, and the older adult population has experienced a marked increase since 2010; this increase in life expectancy has been higher for men $^{(1)}$ in such a way that if it remains so, it is estimated that more than $35 \%$ of the population will be older adults by 2070 . As a result of this phenomenon of population aging, a significant increase may occur in the prevalence of chronic non-communicable diseases, with an emphasis on those related to low bone mineral density (BMD) (osteopenia and osteoporosis), as well as its consequences such as the risk of falls and fractures. It is estimated that the risk of fractures due to low bone mass levels from the age of 40 is $25.6 \%$ in women and $15.5 \%$ in men, with hip, distal radius and vertebrae (compression fracture) becoming more common, along with a higher incidence in older postmenopausal white women ${ }^{(2)}$. On the other hand, there has been a significant increase in hip fractures in men, with about $30 \%$ of total hip fractures occurring in this group and half of them before the age of 80 years $^{(3)}$.

There is a large variation between the results of studies on the prevalence of osteoporosis in Brazil, as they use different methods to evaluate the presence of osteoporosis such as self-report and bone densitometry examination ${ }^{(3-4)}$. Furthermore, most of the studies show no population-based data and are essentially performed with postmenopausal women ${ }^{(5-6)}$. However, a high incidence of osteoporosis has been observed in men in the last 20 years ${ }^{(3)}$. Thus, it is estimated that the general prevalence of osteoporosis in Brazilians ranges from $15 \%$ to $30 \%$ in women ${ }^{(7)}$, and 2 to $8 \%$ in men $^{(3)}$.

In view of this epidemiological situation, the identification of factors associated with bone health is fundamental to combat the progression of osteometabolic diseases. In this sense, studies have shown that age and gender ${ }^{(5-6,8-10)}$, use of medications and hormones ${ }^{(11-12)}$, body composition ${ }^{(6-10)}$, behavioral and environmental aspects ${ }^{(13-14)}$, and food consumption $^{(15)}$ are factors associated with bone metabolism. However, few studies have been conducted with adults of both genders ${ }^{(4,16)}$, and the existing studies assess risk factors in isolation ${ }^{(5,15)}$. Most studies are with older individuals and menopausal women. Studies evaluating bone mineral density in adults are scarce.

Therefore, identification of possible factors associated with the compromise of bone health in adults of both genders is important to understand the process of bone deterioration due to aging. This diagnosis has also become important for men because of the increased incidence of osteoporosis in these individuals. Furthermore, preventive actions may be planned from the identification of such factors in order to mitigate the consequences of declining bone health and public health expenditures. Therefore, the objective of the present study was to evaluate the factors associated with bone mineral density of the lumbar spine (BMD-LS), femoral neck (BMD-FN) and total femur (BMD-TF) in adults.
We hypothesize that sociodemographic, anthropometric, and behavioral factors of health conditions are associated with bone health between men and women.

\section{METHOD}

\section{Study Design}

This is a cross-sectional, population-based study carried out in the period of 2012-2014.

\section{Population}

Subjects of both genders with age ranging from 20 to 59 at the moment of the study, being residents of the urban area of the municipality of Viçosa/MG were considered eligible for participation in this study. The exclusion criteria were pregnant and postpartum women, bedridden individuals, as well as those who had undergone orthopedic surgery, used some type of prosthesis or those who used some medicine that had effect on bone metabolism (corticosteroids, calcium, and vitamin $\mathrm{D}$ supplementation).

The sample was selected by probability. The sampling process was by two-stage conglomerates, with the first-stage units being the census sectors and the second-stage units the households. We performed a sample calculation for this study based on the following parameters: unknown prevalence estimated at 50\%; sample error of 5\%; effect of the conglomerate design estimated at 1.50; and estimated loss percentage at $10 \%$, and this same value for adjustment for confounding factors. Thus, it was determined that the minimum sample required for this research would be 651 individuals.

The average number of residents per household is equivalent to four individuals. There are 300 households multiplied by four people (on average) in each of the census tracts of $\mathrm{Viçosa} / \mathrm{MG}$, equivalent to 1,200 people per census tract or 720 people $(60 \%)$ in the age group of interest for the study. As the required number of the calculated sample was equal to 651 individuals, we then divided this number by the number of drawn census tracts ( 30 sectors) to obtain 22 people between the ages of 20 and 59 required for the study.

\section{Data collection}

Data collection was carried out in three stages. The first stage occurred in the volunteers' residence, in which a semi-structured questionnaire was applied by trained interviewers to collect information on: gender (men and women); age range (20-29, 30-39, 40-49 and 50-59 years); self-declared race/color, according to the classification of the Brazilian population census (white, brown, black, yellow (oriental), and indigenous) $)^{(17)}$ and grouped into whites and non-whites. The 25 participants who declared themselves to be yellow or indigenous were excluded from the analyses because of their low frequency. Information was also collected on: consumption class (high, intermediate and low); years of study completed at the time of the study $(0-4,5-8,9-11$ and $>12$ years); menopause, contraceptive use and hormone replacement therapy (all categorized as yes or no); alcoholism 
(divided into three categories according to the weekly consumption of alcoholic beverages, being $0,1-7$ and > 8 drinks); and smoking (non-smokers, smokers and ex-smokers).

The second stage consisted of evaluation of anthropometric measures, physical activity level (PAL), and blood collection, all performed by trained professionals in the laboratory. Measurements of total body mass $(\mathrm{Kg})$ and height $(\mathrm{cm})$ were obtained according to recommendations in the literature in order to calculate body mass index (BMI), which was calculated using the formula: weight $(\mathrm{kg}) /[\text { height }(\mathrm{m})]^{2(18)}$, and categorized into: eutrophic $\left(\leq 24.9 \mathrm{~kg} / \mathrm{m}^{2}\right)$, overweight $\left(25.0 \mathrm{~kg} / \mathrm{m}^{2}\right.$ to $\left.29.9 \mathrm{~kg} / \mathrm{m}^{2}\right)$, and obese $\left(\geq 30.0 \mathrm{~kg} / \mathrm{m}^{2}\right)^{(18)}$.

PAL was determined through the International Physical Activity Questionnaire (IPAQ), version 6, long format, validated for the Brazilian population ${ }^{(19)}$. PAL score was calculated by summing the time spent doing physical activity (PA) of moderate intensity (including walking) and vigorous intensity, which was obtained by the time spent doing vigorous $\mathrm{PA}$ multiplied by two $[(\mathrm{PAL}=$ moderate $\mathrm{PA}+$ (vigorous $\mathrm{PAx} 2)]$. The PAL of the individuals evaluated was subsequently calculated according to Domain 4 in the IPAQ, referring to recreational, sports, exercise and leisure physical activities. PAL was categorized according to the time of PA performed in the week prior to applying the questionnaire into: irregularly active (IA) $(<150 \mathrm{~min} /$ week $)$, and physically active (PA) $(\geq 150 \mathrm{~min} / \text { week })^{(19)}$.

Blood collection was performed to determine the 25 Hidroxyvitamin D [25(OH)D] status, which was evaluated by chemiluminescence, using the ARCHITECT 25(OH) $\mathrm{D}$ kit, on the ARCHITECT/ABBOTT instrument. Blood samples were collected by peripheral endovenous puncture using the disposable vacuum system after 12 hours of fasting between seven and ten o'clock in the morning. The $25(\mathrm{OH})$ $\mathrm{D}$ status was determined according to the following reference values: sufficient $(\geq 30.0 \mathrm{ng} / \mathrm{ml})$, insufficient $(21.0 \mathrm{ng} /$ $\mathrm{ml}$ to $29.9 \mathrm{ng} / \mathrm{ml})$, and deficient $(\leq 20.9 \mathrm{ng} / \mathrm{ml})^{(20)}$. Although there is a new proposal for the $25(\mathrm{OH}) \mathrm{D}$ reference intervals for the Brazilian population, we decided to use the current classification during data collection.

The last stage of data collection comprises the evaluation of the dependent variable of this study, BMD, using the dual-energy x-ray absorptiometry (DXA) Lunar Prodigy Advance DXA System-GE Healthcare. Accuracy and safety were assessed on the DXA before the exams, and BMD-LS, BMD-FN and BMD-TH were mapped with the individual in dorsal decubitus position. All participants were evaluated on the same DXA equipment. The results were compiled and presented to the volunteers in a report issued by the physician in charge so that those who presented changes received recommendations to seek out a specialist. The absolute values of BMD expressed as $\mathrm{g} / \mathrm{cm}^{2}$ were considered for statistical analyses.

\section{ANALYSIS AND PROCESSING OF DATA}

Data were double-typed in the Epidata program, version 3.1. After testing their consistency, statistical analyzes were performed in Stata 13.1. The analysis was weighted by gender, age and schooling, and the weights were determined by the ratio between the proportions of individuals in the sample ${ }^{(17)}$. The descriptive analysis was performed from the calculation of proportions, means and their respective 95\% confidence intervals (95\% CI). Differences between the groups were verified through the comparison of the $95 \%$ CIs. The normality of the dependent variable was tested using the Shapiro-Wilk test and histograms. The association between BMD and the independent variables was evaluated by simple and multiple linear regression analysis. Variables with $p$-value $\leq 0.25$ in the simple regression analysis were tested in the multiple models. Only the variables with $p$-value $\leq 0.05$ were maintained in the final model. The significance level adopted for all analyses was $5 \%(\alpha=0.05)$, and stratified by gender.

\section{ETHICAL ASPECTS}

This study is part of a larger research project, approved by the Ethics Committee on Human Research of the Universidade Federal de Viçosa (No. 02/2013/ $\mathrm{CEP} / 12.07 .2013)$. All volunteers who agreed to participate signed the informed consent form in accordance with the National Health Council's Regulatory Guidelines for Research Involving Human Beings, Ministry of Health ${ }^{(21)}$. The return was sent with the results to all study participants. Those who presented alterations in one of the behavioral, biochemical and/or anthropometric variables, or in relation to bone health were asked to look for qualified professionals or the health unit closest to their residence, and were informed of their location.

\section{RESULTS}

From the total of 701 individuals included in the study, $50.3 \%$ were female, $26.2 \%$ were between 30 and 39 years old, $61.4 \%$ declared themselves to be non-white, $65.5 \%$ belonged to the intermediate consumption class, and $42.8 \%$ had high school education, with more than 12 completed years of study. The sample presented $50.3 \%$ eutrophic individuals, $72.6 \%$ IA individuals, $65.1 \%$ non-smokers, and $46.0 \%$ did not consume alcoholic beverages. Regarding the $25(\mathrm{OH})$ D status, $49.0 \%$ were classified as having sufficient levels. In relation to women, $64.7 \%$ were non-menopausal, $96.4 \%$ did not use hormone replacement, and $81.0 \%$ used contraceptives. Men and women were similar in relation to the distribution of study variables, except that men reported higher consumption of alcoholic beverages and women had a higher frequency of 25(OH)D deficiency.

Table 1 shows the mean values of BMD-LS, BMD-FN, and BMD-TH according to the study variables in men. BMD values were significantly higher among obese than eutrophic individuals for all evaluated bone sites. In addition, mean BMD-LS and BMD-TH were higher in the overweight compared to the eutrophic individuals. On the other hand, significantly lower bone mass values only for the BMD-FN site were observed among older individuals (40-49 and 50-59 years old) than among younger ones (20-29 years old), and among the less educated (0-4 years of schooling) compared to those with higher education (9-11 and $\geq 12$ years). Men with $25(\mathrm{OH}) \mathrm{D}$ deficiency had 
bone mass means which were significantly lower than those individuals with $25(\mathrm{OH}) \mathrm{D}$ insufficiency had a higher mean with sufficiency, regardless of the site evaluated. In addition, of BMD-TH than those with deficiency (Table 1).

Table 1 - Mean values, BMD confidence intervals according to study variables among men - Viçosa, MG, Brazil, 2012-2014. (n=701)

\begin{tabular}{|c|c|c|c|c|c|c|}
\hline \multirow{2}{*}{ Variables } & \multicolumn{2}{|c|}{ BMD-LS (g/cm²) } & \multicolumn{2}{|c|}{ BMD-FN $\left(\mathrm{g} / \mathrm{cm}^{2}\right)$} & \multicolumn{2}{|c|}{ BMD-TH $\left(\mathrm{g} / \mathrm{cm}^{2}\right)$} \\
\hline & Mean & $\mathrm{Cl} 95 \%$ & Mean & $\mathrm{Cl} 95 \%$ & Mean & $\mathrm{Cl} 95 \%$ \\
\hline Overall & \multicolumn{2}{|c|}{$1.204(1.181-1.227)$} & \multicolumn{2}{|c|}{$1.094(1.075-1.113)$} & \multicolumn{2}{|c|}{$1.093(1.079-1.106)$} \\
\hline \multicolumn{7}{|c|}{ Age range (years) } \\
\hline $20-29$ & 1.220 & $1.194-1.246$ & $1.154^{\mathrm{a}}$ & $1.131-1.176$ & 1.120 & $1.098-1.143$ \\
\hline $30-39$ & 1.234 & $1.193-1.275$ & $1.107^{b}$ & $1.075-1.140$ & 1.086 & $1.055-1.118$ \\
\hline $40-49$ & 1.182 & $1.126-1.239$ & $1.073^{\mathrm{a}}$ & $1.039-1.106$ & 1.081 & $1.049-1.113$ \\
\hline $50-59$ & 1.168 & $1.116-1.221$ & $1.025^{\mathrm{a}, \mathrm{b}}$ & $0.982-1.068$ & 1.076 & $1.034-1.119$ \\
\hline \multicolumn{7}{|l|}{ Skin Color } \\
\hline White & 1.174 & $1.137-1.211$ & 1.074 & $1.046-1.102$ & 1.073 & $1.051-1.095$ \\
\hline Non-white & 1.226 & $1.197-1.255$ & 1.109 & $1.083-1.136$ & 1.107 & $1.083-1.130$ \\
\hline \multicolumn{7}{|c|}{ Consumption Class } \\
\hline High & 1.211 & $1.175-1.248$ & 1.097 & $1.061-1.134$ & 1.098 & $1.072-1.124$ \\
\hline Intermediate & 1.201 & $1.170-1.231$ & 1.094 & $1.074-1.113$ & 1.090 & $1.073-1.106$ \\
\hline Low & 1.194 & $1.123-1.264$ & 1.089 & $1.031-1.147$ & 1.102 & $1.043-1.162$ \\
\hline \multicolumn{7}{|c|}{ Education (years) } \\
\hline $0-4$ & 1.195 & $1.170-1.220$ & $1.041^{\mathrm{a}}$ & $0.998-1.085$ & 1.095 & $1.064-1.126$ \\
\hline $5-8$ & 1.171 & $1.103-1.239$ & 1.072 & $1.023-1.121$ & 1.077 & $1.037-1.118$ \\
\hline $9-11$ & 1.230 & $1.181-1.279$ & $1.127^{\mathrm{a}}$ & $1.089-1.165$ & 1.127 & $1.093-1.161$ \\
\hline$\geq 12$ & 1.207 & $1.172-1.242$ & $1.107^{\mathrm{a}}$ & $1.086-1.128$ & 1.083 & $1.064-1.102$ \\
\hline \multicolumn{7}{|c|}{ Nutritional Status } \\
\hline Eutrophic & $1.159^{\mathrm{a}}$ & $1.128-1.191$ & $1.070^{\mathrm{a}}$ & $1.040-1.099$ & $1.053^{\mathrm{a}}$ & $1.030-1.077$ \\
\hline Overweight & $1.246^{\mathrm{a}}$ & $1.209-1.283$ & 1.102 & $1.068-1.137$ & $1.111^{\mathrm{a}, \mathrm{b}}$ & $1.085-1.137$ \\
\hline Obese & $1.244^{\mathrm{a}}$ & $1.197-1.290$ & $1.163^{\mathrm{a}}$ & $1.110-1.215$ & $1.183^{a, b}$ & $1.142-1.225$ \\
\hline \multicolumn{7}{|l|}{ PAL } \\
\hline $\mathrm{IA}$ & 1.192 & $1.170-1.215$ & 1.080 & $1.059-1.100$ & 1.086 & $1.070-1.103$ \\
\hline $\mathrm{PA}$ & 1.233 & 1.176-1.290 & 1.135 & $1.098-1.172$ & 1.111 & $1.076-1.146$ \\
\hline \multicolumn{7}{|l|}{ Smoking } \\
\hline Non-smoker & 1.214 & $1.184-1.244$ & 1.107 & $1.089-1.124$ & 1.089 & $1.074-1.104$ \\
\hline Smoker & 1.178 & 1.138-.1.219 & 1.084 & $1.041-1.128$ & 1.096 & $1.054-1.138$ \\
\hline Ex-smoker & 1.192 & $1.150-1.235$ & 1.068 & $1.032-1.105$ & 1.101 & $1.073-1.128$ \\
\hline \multicolumn{7}{|c|}{ Alcoholism (drinks/week) } \\
\hline 0 & 1.200 & $1.160-1.240$ & 1.072 & $1.023-1.121$ & 1.079 & $1.043-1.115$ \\
\hline $1-7$ & 1.203 & $1.170-1.235$ & 1.104 & $1.080-1.128$ & 1.088 & $1.065-1.111$ \\
\hline 8 or more & 1.211 & $1.173-1.249$ & 1.104 & $1.074-1.134$ & 1.120 & $1.097-1.144$ \\
\hline \multicolumn{7}{|l|}{$25(\mathrm{OH}) \mathrm{D}$} \\
\hline Sufficient & $1.219^{\mathrm{a}}$ & $1.195-1.243$ & $1.109^{\mathrm{a}}$ & $1.086-1.132$ & $1.106^{\mathrm{a}}$ & $1.090-1.122$ \\
\hline Insufficient & 1.205 & $1.163-1.247$ & 1.090 & $1.050-1.130$ & 1.095 & $1.061-1.128$ \\
\hline Deficient & $1.104^{\mathrm{a}}$ & $1.038-1.170$ & $1.020^{\mathrm{a}}$ & $0.965-1.074$ & $1.002^{\mathrm{a}}$ & $0.945-1.059$ \\
\hline
\end{tabular}

BMD-LS: lumbar spine mineral density; BMD-FN: femoral neck mineral density; BMD-TH: total hip mineral density; PAL: physical activity level; IA: irregularly active; PA: physically active; 25(OH)D: 25-Hydroxyvitamin D. Same letters indicate statistically significant difference.

Table 2 shows the mean values of BMD according to the study variables in the three evaluated bone sites among women. Significantly lower values of BMD-LS and BMD-FN were observed among older women (50-59 years old) compared with younger women (20-29 and 30-39 years old). Regarding nutritional status, obese women also presented significantly higher mean of BMD-FN and BMD-TH than those with overweight and eutrophic. Finally, higher bone mass was identified in the BMD-LS and BMD-FN sites among those non-menopausal and who did not use contraceptives, respectively. When comparing mean BMD between genders, men presented significantly higher values than women for all evaluated bone sites (Table 1 and 2).

Tables 3 and 4 show the gross and adjusted associations of the independent variables which remained in the final model with BMD according to gender. The variables age range, nutritional status, and 25(OH)D for men were significantly associated with BMD in all evaluated bone sites. In addition, race/color was also associated with $\mathrm{BMD}$, however, only in the BMD-LS and DMO-FN bone sites. In the adjusted models we identified that BMD is lower in older individuals, in those who are overweight and obese, non-white, and among those with deficient levels of 25(OH)D (Table 3). We also identified variations in the final models in relation to the evaluated bone sites for women. The variables age range and nutritional status were significantly associated with BMD, regardless of the bone site evaluated, with lower bone mass among older individuals and among eutrophic individuals. Higher bone mass was found among non-white compared to white patients in the BMD-LS and BMD-FN bone sites. Finally, an association was found between using contraceptives and lower BMD-LS (Table 4). 
Table 2 - Mean values and confidence intervals (95\% Cl) of lumbar spine and femur BMD according to study variables among women - Viçosa, MG, Brazil, 2012-2014. ( $n=701)$

\begin{tabular}{|c|c|c|c|c|c|c|}
\hline \multirow{2}{*}{ Variables } & \multicolumn{2}{|c|}{ BMD-LS (g/cm²) } & \multicolumn{2}{|c|}{ BMD-FN $\left(\mathrm{g} / \mathrm{cm}^{2}\right)$} & \multicolumn{2}{|c|}{ BMD-TH $\left(\mathrm{g} / \mathrm{cm}^{2}\right)$} \\
\hline & Mean & Cl $95 \%$ & Mean & Cl $95 \%$ & Mean & Cl $95 \%$ \\
\hline Overall & \multicolumn{2}{|c|}{$1.151(1.131-1.171)$} & \multicolumn{2}{|c|}{$0.991(0.974-1.007)$} & \multicolumn{2}{|c|}{$0.999(0.981-1.017)$} \\
\hline \multicolumn{7}{|c|}{ Age range (years) } \\
\hline $20-29$ & $1.186^{\mathrm{a}}$ & $1.161-1.210$ & $1.032^{\mathrm{a}}$ & $1.009-1.054$ & 1.019 & $0.996-1.042$ \\
\hline $30-39$ & $1.200^{\mathrm{b}}$ & $1.159-1.241$ & $1.031^{\mathrm{b}}$ & $0.996-1.065$ & 1.010 & $0.970-1.051$ \\
\hline $40-49$ & $1.184^{c}$ & $1.156-1.212$ & 0.987 & $0.958-1.016$ & 1.007 & $0.973-1.040$ \\
\hline $50-59$ & $1.055^{\mathrm{a}, \mathrm{b}, \mathrm{c}}$ & $1.005-1.104$ & $0.926^{a, b}$ & $0.889-0.963$ & 0.967 & 0.934-1.001 \\
\hline \multicolumn{7}{|l|}{ Skin Color } \\
\hline White & 1.115 & $1.085-1.144$ & 0.965 & 0.939-0.992 & 0.975 & $0.952-0.998$ \\
\hline Non-white & 1.170 & $1.142-1.197$ & 1.004 & $0.982-1.026$ & 1.013 & $0.986-1.039$ \\
\hline \multicolumn{7}{|c|}{ Consumption Class } \\
\hline High & 1.156 & $1.120-1.191$ & 0.980 & $0.955-1.006$ & 0.980 & $0.945-1.014$ \\
\hline Intermediate & 1.155 & $1.131-1.179$ & 0.993 & $0.971-1.015$ & 1.003 & $0.982-1.023$ \\
\hline Low & 1.121 & $1.046-1.195$ & 0.997 & $0.942-1.053$ & 1.019 & $0.957-1.081$ \\
\hline \multicolumn{7}{|c|}{ Education (years) } \\
\hline $0-4$ & 1.116 & $1.047-1.186$ & 0.963 & $0.920-1.006$ & 0.995 & $0.951-1.039$ \\
\hline $5-8$ & 1.159 & $1.111-1.207$ & 1.018 & $0.977-1.059$ & 1.028 & $0.990-1.066$ \\
\hline $9-11$ & 1.145 & $1.109-1.182$ & 0.991 & $0.961-1.022$ & 0.992 & $0.958-1.025$ \\
\hline$\geq 12$ & 1.172 & $1.151-1.194$ & 0.994 & $0.971-1.017$ & 0.993 & $0.971-1.016$ \\
\hline \multicolumn{7}{|c|}{ Nutritional Status } \\
\hline Eutrophic & 1.136 & $1.113-1.160$ & $0.972^{\mathrm{a}}$ & $0.949-0.994$ & $0.966^{\mathrm{a}}$ & $0.942-0.990$ \\
\hline Overweight & 1.157 & $1.120-1.193$ & $0.983^{b}$ & $0.957-1.010$ & $0.999^{b}$ & $0.973-1.025$ \\
\hline Obese & 1.181 & $1.145-1.217$ & $1.050^{a, b}$ & $1.012-1.089$ & $1.087^{a, b}$ & $1.060-1.114$ \\
\hline \multicolumn{7}{|l|}{ PAL } \\
\hline IA & 1.148 & $1.121-1.175$ & 0.998 & $0.976-1.019$ & 1.001 & $0.979-1.024$ \\
\hline PA & 1.158 & $1.126-1.190$ & 0.972 & $0.942-1.003$ & 0.994 & $0.964-1.024$ \\
\hline \multicolumn{7}{|l|}{ Smoking } \\
\hline Non-smoker & 1.163 & $1.136-1.190$ & 1.000 & $0.977-1.022$ & 1.003 & $0.982-1.024$ \\
\hline Smoker & 1.115 & $1.058-1.172$ & 0.968 & $0.929-1.007$ & 0.979 & $0.942-1.015$ \\
\hline Ex-smoker & 1.130 & $1.096-1.163$ & 0.972 & $0.938-1.006$ & 0.997 & $0.958-1.036$ \\
\hline \multicolumn{7}{|c|}{ Alcoholism (drinks/week) } \\
\hline 0 & 1.141 & $1.117-1.165$ & 0.985 & $0.964-1.006$ & 1.000 & $0.982-1.018$ \\
\hline $1-7$ & 1.162 & $1.125-1.200$ & 0.993 & $0.967-1.019$ & 0.990 & $0.959-1.020$ \\
\hline 8 or more & 1.206 & $1.160-1.252$ & 1.053 & $0.989-1.116$ & 1.065 & $1.016-1.115$ \\
\hline \multicolumn{7}{|l|}{ Menopause } \\
\hline No & $1.189^{\mathrm{a}}$ & $1.163-1.215$ & 1.016 & $0.955-1.037$ & 1.011 & $0.987-1.035$ \\
\hline Yes & $1.081^{\mathrm{a}}$ & $1.034-1.128$ & 0.942 & $0.909-0.975$ & 0.976 & $0.945-1.008$ \\
\hline \multicolumn{7}{|c|}{ Hormone Replacement Therapy } \\
\hline No & 1.152 & $1.132-1.171$ & 0.992 & $0.975-1.010$ & 1.000 & $0.981-1.019$ \\
\hline Yes & 1.130 & $1.028-1.233$ & 0.943 & $0.887-0.998$ & 0.982 & $0.924-1.041$ \\
\hline \multicolumn{7}{|l|}{ Contraceptive } \\
\hline No & 1.189 & $1.164-1.214$ & $1.033^{\mathrm{a}}$ & $1.005-1.060$ & 1.020 & $0.991-1.048$ \\
\hline Yes & 1.140 & $1.111-1.168$ & $0.976^{\mathrm{a}}$ & $0.954-0.998$ & 0.966 & $0.974-1.017$ \\
\hline \multicolumn{7}{|l|}{$25(\mathrm{OH}) \mathrm{D}$} \\
\hline Sufficient & 1.168 & $1.138-1.198^{a}$ & $0.991^{\mathrm{a}}$ & $0.969-1.013$ & 0.989 & $0.961-1.017$ \\
\hline Insufficient & 1.136 & $1.101-1.171$ & 0.976 & $0.951-1.000$ & 0.966 & $0.970-1.021$ \\
\hline Deficient & 1.145 & $1.098-1.193^{\mathrm{a}}$ & $1.022^{\mathrm{a}}$ & $0.974-1.069$ & 1.032 & $0.993-1.071$ \\
\hline
\end{tabular}

BMD-LS: lumbar spine mineral density; BMD-FN: femoral neck mineral density; BMD-TH: total hip mineral density; PAL: physical activity level; IA: irregularly active; PA: physically active; 25(OH)D:25-Hydroxyvitamin D. Same letters indicate statistically significant differences. 
Table 3 - Simple, multiple linear regression, confidence intervals, p-value for BMD among men - Viçosa, MG, Brazil, 2012-2014. ( $\mathrm{n}=701$ )

\begin{tabular}{|c|c|c|c|c|c|c|c|c|c|c|c|c|}
\hline \multirow{2}{*}{ Variables } & \multicolumn{4}{|c|}{ BMD-LS $\left(\mathrm{g} / \mathrm{cm}^{2}\right)$} & \multicolumn{4}{|c|}{ BMD-FN $\left(\mathrm{g} / \mathrm{cm}^{2}\right)$} & \multicolumn{4}{|c|}{ BMD-TH $\left(\mathrm{g} / \mathrm{cm}^{2}\right)$} \\
\hline & B & $\mathrm{Cl} 95 \%$ & $B A j$ & $\mathrm{Cl} 95 \%$ & B & $\mathrm{Cl} 95 \%$ & $B A j$ & $\mathrm{Cl} 95 \%$ & B & $\mathrm{Cl} 95 \%$ & $\mathrm{BAj}$ & $\mathrm{Cl} 95 \%$ \\
\hline \multicolumn{13}{|c|}{ Age Range (years) } \\
\hline $20-29$ & 0.00 & --- & 0.00 & --- & 0.00 & --- & 0.00 & --- & 0.00 & --- & --- & --- \\
\hline $30-39$ & 0.14 & $-0.03 ; 0.06$ & 0.06 & $-0.03 ; 0.05$ & -0.04 & $-0.08 ;-0.09$ & -0.05 & $-0.08 ;-0.02$ & 0.03 & $-0.06 ; 0.01$ & -0.04 & $-0.07 ;-0.01$ \\
\hline $40-49$ & -0.03 & $-0.09 ; 0.02$ & -0.07 & $-0.11 ;-0.02$ & -0.08 & $-0.12 ;-0.03$ & -0.10 & $-0.14 ;-0.06$ & -0.04 & $-0.09 ; 0.02$ & -0.10 & $-0.09 ;-0.06$ \\
\hline $50-59$ & -0.05 & $-0.10 ;-0.06$ & -0.07 & $-0.12 ;-0.02$ & -0.12 & $-0.17 ;-0.07$ & -0.15 & $-0.19 ;-0.11$ & -0.04 & $-0.09 ; 0.04$ & -0.15 & $-0.14 ;-0.11$ \\
\hline
\end{tabular}

Skin Color

$\begin{array}{lcccccccc}\text { White } & 0.00 & --- & 0.00 & --- & 0.00 & --- & 0.00 & --- \\ \text { Non-White } & 0.05 & 0.04 ; 0.09 & 0.03 & 0.02 ; 0.07 & 0.03 & -0.04 ; 0.07 & 0.03 & 0.00 ; 0.06\end{array}$

\begin{tabular}{|c|c|c|c|c|c|c|c|c|c|c|c|c|}
\hline \multicolumn{13}{|c|}{ Nutritional Status } \\
\hline Eutrophic & 0.00 & --- & 0.00 & --- & 0.00 & --- & 0.00 & --- & 0.00 & --- & --- & --- \\
\hline Overweight & 0.08 & $0.03 ; 0.13$ & 0.10 & $0.05 ; 0.14$ & 0.03 & $-0.01 ; 0.08$ & 0.05 & $0.07 ; 0.10$ & 0.05 & $0.01 ; 0.09$ & 0.06 & $0.01 ; 0.10$ \\
\hline Obese & 0.08 & $0.03 ; 0.13$ & 0.10 & $0.05 ; 0.14$ & 0.09 & $0.03 ; 0.15$ & 0.12 & $0.07 ; 0.18$ & 0.13 & $0.08 ; 0.17$ & 0.13 & $0.07 ; 0.19$ \\
\hline \multicolumn{13}{|l|}{$25(\mathrm{OH}) \mathrm{D}$} \\
\hline Sufficient & 0.00 & --- & 0.00 & --- & 0.00 & --- & 0.00 & --- & 0.00 & --- & --- & --- \\
\hline Insufficient & -0.01 & $-0.06 ; 0.03$ & -0.02 & $-0.06 ; 0.02$ & -0.01 & $-0.06 ; 0.02$ & -0.01 & $-0.05 ; 0.18$ & -0.01 & $-0.04 ; 0.02$ & -0.01 & $-0.04 ; 0.02$ \\
\hline Deficient & -0.11 & $-0.18 ;-0.04$ & -0.09 & $-0.16 ;-0.05$ & -0.08 & $-0.14 ;-0.03$ & -0.08 & $-0.01 ;-0.03$ & -0.01 & $-0.06 ;-0.04$ & -0.08 & $-0.13 ;-0.04$ \\
\hline
\end{tabular}

BMD-LS: bone mineral density of the lumbar spine; BMD-FN: bone mineral density of the femoral neck; BMD-TH: bone mineral density of total hip.

ß: $ß$ value for simple linear regression

BAdj: $ß$ value adjusted to covariates

Table 4 - Simple, multiple linear regression, confidence intervals, p-value for BMD among women - Viçosa, MG, Brazil, 2012-2014. (n=701)

\begin{tabular}{|c|c|c|c|c|c|c|c|c|c|c|c|c|}
\hline \multirow{2}{*}{ Variable } & \multicolumn{4}{|c|}{ BMD-LS $\left(\mathrm{g} / \mathrm{cm}^{2}\right)$} & \multicolumn{4}{|c|}{ BMD-FN (g/cm²) } & \multicolumn{4}{|c|}{ BMD-TH $\left(\mathrm{g} / \mathrm{cm}^{2}\right)$} \\
\hline & B & Cl 95\% & $B A j$ & $\mathrm{Cl} 95 \%$ & B & $\mathrm{Cl} 95 \%$ & $B A j$ & $\mathrm{Cl} 95 \%$ & B & $\mathrm{Cl} 95 \%$ & $\mathrm{BAj}$ & Cl 95\% \\
\hline \multicolumn{13}{|c|}{ Age Range (years) } \\
\hline $20-29$ & 0.00 & -- & 0.00 & --- & 0.00 & --- & 0.00 & --- & 0.00 & --- & 0.00 & --- \\
\hline $30-39$ & 0.14 & $-0.03 ; 0.06$ & 0.01 & $-0.05 ; 0.07$ & -0.01 & $-0.03 ; 0.03$ & -0.01 & $-0.05 ; 0.01$ & -0.08 & $-0.04 ; 0.02$ & -0.02 & $-0.06 ; 0.08$ \\
\hline $40-49$ & -0.01 & $-0.04 ; 0.03$ & -0.03 & $-0.08 ; 0.02$ & -0.04 & $-0.08 ;-0.03$ & -0.07 & $-0.11 ;-0.03$ & -0.01 & $-0.05 ; 0.03$ & -0.04 & $-0.08 ;-0.03$ \\
\hline $50-59$ & -0.13 & $-0.19 ;-0.07$ & -0.16 & $-0.23 ;-0.10$ & -0.10 & $-0.14 ;-0.06$ & -0.13 & $-0.17 ;-0.09$ & -0.05 & $-0.08 ;-0.01$ & -0.09 & $-0.13 ;-0.05$ \\
\hline \multicolumn{13}{|l|}{ Skin Color } \\
\hline White & 0.00 & --- & 0.00 & --- & 0.00 & --- & 0.00 & --- & \multirow{2}{*}{\multicolumn{4}{|c|}{---}} \\
\hline Non-White & 0.05 & $0.01 ; 0.09$ & 0.04 & $0.09 ; 0.08$ & 0.03 & $0.04 ; 0.07$ & 0.02 & $-0.01 ; 0.05$ & & & & \\
\hline \multicolumn{13}{|l|}{ Contraceptive } \\
\hline No & 0.00 & --- & 0.00 & --- & \multirow{2}{*}{\multicolumn{8}{|c|}{--- }} \\
\hline Yes & 0.04 & $0.06 ; 0.09$ & -0.01 & $-0.06 ; 0.02$ & & & & & & & & \\
\hline \multicolumn{13}{|c|}{ Nutritional Status } \\
\hline Eutrophic & 0.00 & --- & 0.00 & --- & --- & --- & --- & --- & 0.00 & --- & 0.00 & --- \\
\hline Overweight & 0.02 & $-0.02 ; 0.06$ & 0.02 & $-0.01 ; 0.06$ & 0.01 & $-0.02 ; 0.04$ & 0.03 & $0.03 ; 0.06$ & 0.03 & $-0.09 ; 0.06$ & 0.04 & $0.01 ; 0.08$ \\
\hline Obese & 0.04 & $-0.06 ; 0.08$ & 0.04 & $0.01 ; 0.07$ & 0.07 & $0.03 ; 0.12$ & 0.10 & $0.07 ; 0.14$ & 0.12 & $0.08 ; 0.15$ & 0.14 & $0.10 ; 0.17$ \\
\hline
\end{tabular}

BMD-LS: bone mineral density of the lumbar spine; BMD-FN: bone mineral density of the femoral neck; BMD-TH: bone mineral density of total hip.

B: $ß$ value for simple linear regression

BAdj: $ß$ value adjusted to covariates

In addition to the variables present in the final models (Tables 3 and 4), the following variables with $p<0.25$ were introduced in the simple linear regression: at the BMD-FN site for men, education ( $p=0.005)$ and PAL $(p=0.017)$; at the BMD-LS site for women, smoking ( $\mathrm{p}=0.053)$, alcoholism $(\mathrm{p}=0.094)$, and menopause $(\mathrm{p}=0.027)$; and at the BMD-FN site for women, menopause $(\mathrm{p}=0.002)$, hormone replacement therapy $(\mathrm{p}=0.098)$, and contraceptive use $(\mathrm{p}=0.003)$. However, these did not remain in the final models, since they were non-significant $(p>0.05)$ in the multiple models.

\section{DISCUSSION}

In this study, we identified that men had significantly higher mean BMD values than women. The main variables associated with BMD of the evaluated bone sites in both genders were age range, race/color, and nutritional status. In addition, associations with $25(\mathrm{OH}) \mathrm{D}$ status were identified among men, and contraceptive use among women.

Bone health is usually evaluated by biophysical parameters which quantify bone mass (bone mineral content and density). However, BMD is more clinically used since it evaluates specific bone sites in which the osteopenia process begins.

We identified that lower BMD values are associated with high age, which corroborates other studies ${ }^{(5,9)}$. Bone loss in both genders is a consequence of the deleterious effects of aging, because the balance between bone formation and absorption changes and BMD progressively decreases as age increases. There is a peak in bone mass and accumulation of bone tissue up to 30 years, such that men accumulate $25 \%$ more BMD than women ${ }^{(22)}$, which contributes to delaying the loss of BMD in men. Then there is a gradual decline which intensifies after menopause for women due 
to a decrease in estrogen, while it occurs for men after 70 years when there is also a decrease in testosterone levels, which delays the consequences of loss of BMD, such as fractures (for example) in relation to women ${ }^{(8)}$. Thus, bone metabolism during adulthood should remain balanced, as this will contribute to good bone health and will lower the risk of bone loss as one ages.

Therefore, bone loss is also related to hormonal issues, with sex hormones being one of the main biological agents regulating bone health and responsible for maturation and sexual dimorphism of the skeleton ${ }^{(22)}$. However, no differences were observed among women who use or do not use hormone replacement therapy in our study, since less than $5 \%$ used it because it is a younger population, and it is known that hormone replacement has been used as a prevention for the decline of bone health during menopause in an attempt to delay the deleterious effects of bone health in women during this period ${ }^{(12)}$.

In addition to the issues mentioned above, behavioral aspects have been pointed out as a possible explanation for the differences in bone health between men and women, among which PA and contraceptive use stand out. Although no association between PAL and bone health has been found in this study, it is known that regular PA is considered an important non-pharmacological alternative for bone health maintenance and prevention of osteometabolic diseases. Furthermore, although there is a consensus in the literature regarding these and other benefits related to PA, there is still a high prevalence of inactive people in Brazil, with important differences between genders. A study carried out with Brazilian adults showed that $62.4 \%$ are insufficiently active, with women (70.1\%) being more sedentary than men (53.3\%), and this situation worsens with aging ${ }^{(23)}$. The non-association between PAL and bone health in this study is attributed to the instrument used for the assessment of PAL, since it has important limitations in that it does not consider the history and type of exercise performed, only frequency and intensity at the current moment ${ }^{(20)}$.

With respect to behavioral factors in addition to PA, there is strong evidence that excessive alcohol consumption and smoking has a negative influence on bone metabolism ${ }^{(13-14)}$. Regarding smoking, we found that smokers and former smokers in our study had lower BMD means than non-smokers, although these differences were not significant. The literature indicates that nicotine directly interferes in the amount of calcium absorbed by the skeleton, on the activity of vitamin $\mathrm{D}$, and consequently of parathyroid hormone, in addition to inducing weight loss and physical inactivity, which may not favor the maintenance of bone health ${ }^{(13-14)}$. However, the results of the studies on the subject are inconsistent, so there is a need to carry out further investigations which consider other aspects such as the time of exposure to tobacco and the amount of cigarettes consumed ${ }^{(24)}$. In turn, the abusive consumption of alcoholic beverages inhibits the activity of vitamin $\mathrm{D}$, parathyroid, androgen and estrogen hormones, and thus affects bone structure maintenance ${ }^{(16)}$. Despite this evidence, we did not identify any association between these two behaviors and BMD in our study.
Nutritional status was also identified as a positively associated factor with BMD in both genders, as reported by other studies $^{(6,10)}$. According to the authors, this association can be explained by the mechanical pressure that the overloaded body exerts on the skeleton stimulating bone metabolism. However, obesity is considered a serious global public health problem, constituting a risk factor for several diseases such as type II diabetes and cardiovascular disease ${ }^{(25)}$. Furthermore, unlike our findings, being overweight was found to lead to metabolic alterations detrimental to bone health such as insulin resistance and overproduction of the hormones androgen and estrogen, which reduce osteoblast activity ${ }^{(25)}$. In addition, excess production of adipokines or leptin levels associated with high intakes of high fat foods contributes to increased intestinal calcium absorption, leading to a reduction in bioavailable calcium for bone remodeling ${ }^{(26)}$. Therefore, this association of excess body weight with bone health should be analyzed with great caution, since the harm provided by obesity outweigh the benefits in relation to the activation of bone metabolism.

Another important issue that has been discussed is the association between excess body fat and vitamin $\mathrm{D}$ deficiency $^{(27)}$. Some authors attribute this association to the nutritional deficiency in obese individuals ${ }^{(27-28)}$, or for physiological reasons, as the adipose tissue has bioreceptors which retain $25(\mathrm{OH}) \mathrm{D}$, decreasing the bioavailability of this vitamin ${ }^{(27)}$. Although $25(\mathrm{OH}) \mathrm{D}$ is not considered a bone biomarker, its adequate levels have been pointed out as a physiological parameter positively associated with bone health ${ }^{(15,28)}$. In this sense, we found a positive association between $25(\mathrm{OH}) \mathrm{D}$ and $\mathrm{BMD}$ for all evaluated bone sites among men. In turn, no relationship was observed among women. It is suggested that this finding can be partially explained by the reference values used for the $25(\mathrm{OH}) \mathrm{D}$ status, since it has been shown that women need higher levels of this vitamin to maintain adequate bone activation patterns ${ }^{(28)}$. Therefore, there is a need for more detailed studies to evaluate the possible interrelationships between body composition, bone health, and vitamin D status.

Regarding skin color, other studies report that non-white people have higher bone mass when compared to the reference population (caucasian whites) ${ }^{(28-29)}$, being in agreement with our findings. The relationship between skin color and $\mathrm{BMD}$ is directly related to $25(\mathrm{OH}) \mathrm{D}$ status, as there is a variation between the minimum levels for activation of bone metabolism in whites $(30 \mathrm{ng} / \mathrm{ml})$ and non-whites (20ng/ml), but there is still no consensus in the literature regarding these values ${ }^{(28-29)}$. A study performed with black Americans aged from 28 to 48 years found that $50 \%$ of the African immigrants evaluated increased the supply of parathyroid hormone and absorption of calcium from the minimum levels of $25(\mathrm{OH}) \mathrm{D}(\leq 20 \mathrm{ng} / \mathrm{ml})$, indicating that these individuals have higher BMD than Caucasians ${ }^{(29)}$. This result corroborates other study findings that this $25(\mathrm{OH})$ $\mathrm{D}$ threshold seems to be adequate for the population of African immigrants in the $\mathrm{USA}^{(30)}$. In addition, non-whites have lower vitamin $\mathrm{D}$ concentrations, which is probably due to the increased skin pigmentation which inhibits the 
production of cholecalciferol, the precursor of $25(\mathrm{OH}) \mathrm{D}$ synthesis $^{(30)}$. Thus, despite having lower levels of $25(\mathrm{OH})$ $\mathrm{D}$, black men and women have higher $\mathrm{BMD}^{(30)}$. Although there is evidence that black individuals have denser bones and lower fracture rates than whites, high concentrations of parathyroid hormones resulting from low concentration levels of $25(\mathrm{OH}) \mathrm{D}$ may have negative skeletal consequences for black populations ${ }^{(30)}$. Therefore, although some studies have found an association between race/white skin color and poorer bone health, further studies are needed to assess other intervening factors such as sun exposure, use of sunscreen, metabolism of vitamin $\mathrm{D}$, and other bone biomarkers.

Although this is a cross-sectional study and as such the identified associations cannot be interpreted as a causal relationship, our results contribute to creating prevention strategies for risk factors related to bone health. As previously mentioned, there are few population-based studies on bone health with the adult population and their associated factors, and no studies with such characteristics have been identified in the literature with the Brazilian population. Our results show the importance of bone health evaluation in adults and that identifying factors associated with BMD can contribute to screening individuals with a higher risk of low bone mass who should be a target for preventive measures, mainly for modifiable factors such as overweight and obesity, as well as vitamin $\mathrm{D}$ deficiency. Another positive aspect is the fact that this study was developed with a representative sample of the population which enables extrapolating the results to other regions with similar population characteristics. A key strength of this study is the fact that it is population-based, in which factors associated with low bone health were evaluated in individuals of the general population, including men. This is important because the majority of the studies evaluated post-menopausal women, individuals with high ages, and those presenting some specific harm. Thus, this study contributes to existing research conducted up to the present moment. The limitation to the study is that the behavioral factors (PAL, smoking, and alcoholism) were only evaluated at one point of time, given the transversal nature of the research.

\section{CONCLUSION}

The findings of this study indicate that the main factors associated with low BMD among men were high age, race/ white skin, eutrophy, and deficient status of $25(\mathrm{OH}) \mathrm{D}$. In addition to the same relationships observed for men in relation to age range, race/skin color, and nutritional status, we also identified an association with contraceptive use for women.

\section{RESUMO}

Objetivo: Este estudo tem como objetivo analisar a associação entre coluna lombar, colo femoral, densidade mineral óssea total do quadril (parâmetro biofísico de avaliação da saúde óssea) e fatores sociodemográficos, antropométricos, comportamentais e de condição de saúde nos adultos brasileiros. Método: Este é um estudo transversal, baseado em população, realizado com indivíduos de ambos os gêneros, com idades entre 20 e 59 anos (n=701). As variáveis dependentes foram avaliadas por Absorciometria com Raios-X de Dupla Energia. As variáveis independentes foram avaliadas por meio de um questionário, avaliação antropométrica e coleta de sangue. A associação entre densidade mineral óssea e variáveis independentes foi avaliada por análise de regressão linear. Todas as análises foram estratificadas por gênero. Resultados: Os homens apresentaram densidade mineral óssea mais alta do que as mulheres. A densidade mineral óssea foi inversamente associada à faixa etária e diretamente associada ao estado nutricional em ambos os gêneros e nos três locais ósseos analisados. Além disso, o estado de deficiência de 25-Hidroxivitamina D entre os homens e o uso de contraceptivos entre as mulheres foram associados a uma densidade mineral óssea mais baixa, e uma associação significativa só foi encontrada com a densidade mineral óssea da coluna lombar nas mulheres. Conclusão: Os fatores associados à saúde óssea entre os homens foram idade, cor da pele, estado nutricional e estado da vitamina D. Para as mulheres, os fatores associados à saúde óssea foram idade, cor da pele, estado nutricional e uso de contraceptivos.

\section{DESCRITORES}

Densidade Óssea; Fatores de Risco; Saúde do Adulto; Estudos Epidemiológicos.

\section{RESUMEN}

Objetivo: Este estudio tiene como fin analizar la asociación entre columna lumbar, cuello femoral, densidad mineral ósea total de la cadera (parámetro biofísico de evaluación de la salud ósea) y factores sociodemográficos, antropométricos, comportamentales y de condición de salud en los adultos brasileños. Método: Este es un estudio transversal, basado en población, llevado a cabo con individuos de ambos géneros, con edades entre 20 y 59 años (n=701). Las variables dependientes fueron evaluadas por Absorciometría con Rayos $\mathrm{X}$ de Doble Energía. Las variables independientes fueron evaluadas mediante un cuestionario, evaluación antropométrica y recolección de sangre. La asociación entre densidad mineral ósea y variables independientes fue valorado por análisis de regresión lineal. Todos los análisis fueron estratificados por género. Resultados: Los varones presentaron densidad mineral ósea más alta que las mujeres. La densidad mineral ósea estuvo inversamente asociada con el rango de edad y directamente asociada con el estado nutritivo en ambos géneros y en los tres sitios óseos analizados. Además, el estado de deficiencia de 25-Hidroxivitamina $\mathrm{D}$ entre los hombres y el uso de contraceptivos entre las mujeres estuvieron asociados con una densidad mineral ósea más baja, y una asociación significativa solo fue encontrada con la densidad mineral ósea de la columna lumbar en las mujeres. Conclusión: Los factores asociados con la salud ósea entre los varones fueron edad, color de la piel, estado nutritivo y estado de la vitamina D. Para las mujeres, los factores asociados con la salud ósea fueron edad, color de la piel, estado nutritivo y uso de contraceptivos.

\section{DESCRIPTORES}

Densidad Ósea; Factores de Riesgo; Salud del Adulto; Estudios Epidemiológicos.

\section{REFERENCES}

1. Instituto Brasileiro de Geografia e Estatística. Síntese de indicadores sociais: uma análise das condições de vida da população brasileira [Internet]. Rio de Janeiro: IBGE; 2016 [citado 2018 jan. 29]. Disponível em: https://biblioteca.ibge.gov.br/visualizacao/livros/liv98965.pdf 
2. Pinheiro MM, Ciconelli RM, Jacques NO, Genaro PS, Martini LA, Ferraz MB. The burden of osteoporosis in Brazil: regional data from fractures in adult men and women - The Brazilian Osteoporosis Study (BRAZOS). Rev Bras Reumatol. 2010;50(2):113-27.

3. Loures MAR, Zerbini CAF, Danowski JS, Pereira RMR, Moreira C, Paula AP, et al. Guidelines of the Brazilian Society of Rheumatology for the diagnosis and treatment of osteoporosis in men. Rev Bras Reumatol. 2017;57 Suppl 2:497-514. DOI: 10.1016/j.rbre.2017.07.003

4. Pinheiro MM, Reis Neto ET, Machado FS, Omura F, Yang JHK, Szenjfeld J, et al. Risk factors for osteoporotic fractures and low bone density in pre and postmenopausal women. Rev Saúde Pública. 2010;44(3):479-85.

5. Ilic Stojanovic O, Vuceljic M, Lazovic M, Gajic M, Radosavljevic N, Nikolic D, et al. Bone mineral density at different sites and vertebral fractures in Serbian postmenopausal women. Climacteric. 2017;20(1):37-43. DOI: 10.1080/13697137.2016.1253054

6. Kang H, Chen YM, Han G, Huang H, Chen WQ, Wang X, et al. Associations of age, BMI, and years of menstruation with proximal femur strength in chinese postmenopausal women: a cross-sectional study. Int J Environ Res Public Health. 2016;13(2):157. DOI: 10.3390/ ijerph13020157

7. Baccarro LF, Conde DM, Costa-Paiva L, Pinto-Neto AM. The epidemiology and management of postmenopausal osteoporosis: a viewpoint from Brasil. Clin Interv Aging. 2015;10:583-91. DOI: 10.2147/CIA.S54614

8. Ohnaka K. Aging and homeostasis. Sex hormones and aging. Clin Clacium. 2017;27(7):947-54. DOI: CliCa1707947954

9. Chin KY, Kamaruddin AA, Low NY, Ima-Nirwana S. Effects of age, sex, and ethnicity on bone health status of the elderly in Kuala Lumpur, Malaysia. Clin Intery Aging. 2016;11:767-73. DOI: 10.2147/CIA.S108772

10. Silva ACV, Rosa MI, Fernandes B, Lumertz S, Diniz RM, Damiani MEFR. Factors associated with osteopenia and osteoporosis in women undergoing bone mineral density test. Rev Bras Reumatol. 2015;55(3):223-8. DOI: 10.1016/j.rbr.2014.08.012

11. Jackowski SA, Baxter-Jones ADG, McLardy AJ, Pierson RA, Rodgers CD. The associations of exposure to combined hormonal contraceptive use on bone mineral content and areal bone mineral density accrual from adolescence to young adulthood: a longitudinal study. Bone Rep. 2015;5:e333-e41. DOI: 10.1016/j.bonr.2015.06.001

12. Popat VB, Calis KA, Kalantaridou SN, Vanderhoof VH, Koziol D, Troendle JF, et al. Bone mineral density in young women with primary ovarian insufficiency: results of a three-year randomized controlled trial of physiological transdermal estradiol and testosterone replacement. J Clin Endocrinol Metab. 2014;99(9):3418-26. DOI: 10.1210/jc.2013-4145

13. Emaus N, Wilsgaard T, Ahmed A. Impacts of body mass index, physical activity, and smoking on femoral bone loss: the Tromsø Study. J Bone Min Res. 2014;29(9):2080-9. DOI: 10.1002/jbmr.2232

14. Shin HY, Kang HC, Lee K, Park SM. Association between the awareness of osteoporosis and the quality of care for bone health among Korean women with osteoporosis. BMC Musculoskelet Disord. 2014;15:334. DOI: 10.1186/1471-2474-15-334

15. Joo NS, Yang SW, Song BC, Yeum KJ. Vitamin A intake, serum vitamin D and bone mineral density: analysis of the Korea National Health and Nutrition Examination Survey (KNHANES, 2008-2011). Nutrients. 2015;7(3):1716-27. DOI: 10.3390/nu7031716

16. Chang HK, Chang DG, Myong JP, Kim JH, Lee SJ, Lee YS, et al. Bone mineral density among Korean females aged 20-50 years: influence of age at menarche (The Korea national Health and Nutrition Examination Survey 2008-2001). Osteoporos Int. 2017;28(7):2129-36. DOI: 10.1007/s00198-017-3997-0

17. Instituto Brasileiro de Geografia e Estatística. Censo Demográfico 2010 [Internet]. Rio de Janeiro: IBGE; 2010 [citado 2018 jan. 29]. Disponível em: https://www.ibge.gov.br/

18. World Health Organization. Obesity: preventing and managing the global epidemic. [Internet]. Geneva: WHO; 2000 [cited 2018 Jan 29]. Available from: http://www.who.int/nutrition/publications/obesity/WHO_TRS_894/en/

19. Pardini R, Matsudo S, Araújo T, Matsudo, V, Andrade E, Braggion G, et al. Validação do questionário internacional de nível de atividade física (IPAQ - versão 6): estudo piloto em adultos jovens brasileiros. Rev Bras Ciên Mov. 2001;9(3):45-51.

20. Holick MF, Binkley NC, Bischoff-Ferrari HA, Gordon CM, Hanley DA, Heaney RP, et al. Evaluation, treatment, and prevention of vitamin D deficiency: an Endocrine Society clinical practice guideline. J Clin Endocrinol Metab. 2011;96(7):1911-30. DOI: 10.1210/jc.2011-0385

21. Brasil. Ministério da Saúde; Conselho Nacional de Saúde. Resolução no 466/12. Dispõe sobre diretrizes e normas regulamentadoras de pesquisas envolvendo seres humanos [Internet]. Brasília; 2012 [citado 2018 jan. 29]. Disponível em: http://conselho.saude.gov.br/ resolucoes/2012/Reso466.pdf

22. Fonseca RMC, Pereira RW, França NM. Bone mineral density and content in adolescent girls. Rev Bras Cineantropom Desempenho Hum. 2011;13(5):354-60. DOI: http://dx.doi.org/10.5007/1980-0037.2011v13n5p354

23. Brasil. Ministério da Saúde; Secretaria de Vigilância em Saúde, Departamento de Doenças e Agravos não transmissíveis e Promoção da Saúde. Vigitel Brasil 2016: vigilância de fatores de risco e proteção para doenças crônicas por inquérito telefônico [Internet]. Brasília; 2016 [citado 2018 jan. 29]. Disponível em: http://bvsms.saude.gov.br/bvs/publicacoes/vigitel_brasil_2016_fatores_risco.pdf

24. Strozyk D, Gress TM, Breitling LP. Smoking and bone mineral density: comprehensive analyses of the third National Health and Nutrition Examination Survey (NHANES III). Arch Osteoporos. 2018;13(1):16. DOI: 10.1007/s11657-018-0426-8

25. Silva AS, Lacerda FV, Mota MPG. Efeito do treinamento aeróbio nos níveis de homocisteína em indivíduos diabéticos do tipo 2 . Rev Bras Med Esporte. 2015;21(4):275-8. DOI: http://dx.doi.org/10.1590/1517-869220152104140828

26. Gower BA, Casazza K. Divergent effects of obesity on bone health. J Clin Densitom. 2013;16(4):450-4. DOI: 10.1016/j.jocd.2013.08.010

27. Schuch NJ, Garcia VC, Martini, LA. Vitamin D and endocrine diseases. Arq Bras Endocrinol Metabol. 2009;53(5):625-33.

28. Demeke T, El-Gawad GA, Osmancevic A, Gillstedt M, Landin-Wilhelmsen K. Lower bone mineral density in Somali women living in Sweden compared with African-Americans. Arch Osteoporos. 2015;10:4. DOI:10.1007/s11657-015-0208-5

29. Thorenson CK, Chung ST, Ricks M, Reynolds JC, Remaley AT, Periwal V, et al. Biochemical and clinical deficiency is uncommon in African immigrants despite a high prevalence of low vitamin D: the Africans in America study. Osteoporos Int. 2015;26(11):2607-15. DOI:10.1007/s00198-015-3169-z 
30. Gutiérrez OM, Farwell WR, Kermah D, Taylor EN. Racial differences in the relationship between vitamin D, bone mineral density, and parathyroid hormone in the National Health and Nutrition Examination Survey. Osteoporos Int. 2011;22(6):1745-53. DOI: 10.1007/ s00198-010-1383-2

Financial support:

Fundação de Amparo à Pesquisa de Minas Gerais (FAPEMIG). Process nº APQ-00296-12. Conselho

Nacional de Desenvolvimento Científico e Tecnológico (CNPq). Process nº 481418/2011-3. Coordenação

de Aperfeiçoamento de Pessoal de Nível Superior (CAPES). Academy of Nutrition and Dietetics

Foundation. Universidade Federal de Viçosa Laboratory, MG. Laboratory Bioclin / Quibasa. 